\title{
Health effects of green tea catechins in overweight and obese men: a randomised controlled cross-over trial
}

\author{
A. L. Brown*, J. Lane, C. Holyoak, B. Nicol, A. E. Mayes and T. Dadd \\ Unilever RED, Colworth Science Park, Sharnbrook, Bedfordshire MK44 1LQ, UK \\ (Received 9 August 2010 - Revised 14 March 2011 - Accepted 29 March 2011 - First published online 7 June 2011)
}

\begin{abstract}
Regular consumption of green tea may be cardioprotective. In the present study we investigated the health effects of dietary supplementation with green tea catechins and the potential modifying effect of the catechol-O-methyltransferase (COMT) Val/Met genotype. Subjects (sedentary males, aged 40-69 years, with BMI $\geq 28$ and $\leq 38 \mathrm{~kg} / \mathrm{m}^{2}$ ) were randomly assigned to consume decaffeinated green tea extract (DGT; $530 \mathrm{mg}$ containing about $400 \mathrm{mg}$ total catechins/capsule, twice daily) and placebo in a complete cross-over design. Ambulatory blood pressure and biomarkers of metabolic function (cholesterol, TAG, glucose and insulin) were measured at weeks 0 and 6 . Although a marked increase in the concentration of plasma epigallocatechin gallate (EGCG), urinary epigallocatechin (EGC) and urinary $4^{\prime}-O-$-methyl EGC was found after DGT treatment, no effect on blood pressure or biomarkers of metabolic function was observed. However, a period $\times$ treatment interaction $(P<0.05)$ was detected for body-weight change. Despite a similar increase in estimated energy intake during intervention period 1 , body weight decreased by 0.64 (SD 2.2 ) $\mathrm{kg}$ and increased by 0.53 (SD 1.9) $\mathrm{kg}$ in the DGT and placebo groups, respectively ( $P=0.025$ ), suggesting a protective effect of green tea catechins on weight gain. Additionally, the COMT Val/Met genotype influenced urinary accumulation of EGC and 4'-O-methyl EGC $(P<0 \cdot 01)$. Mean concentrations were lower in individuals homozygous for the high-activity G-allele, possibly reflecting increased metabolic flux and a more rapid conversion to downstream metabolic species, compared with individuals carrying at least one copy of the low-activity A-allele. Additional studies are needed to confirm these findings and further explore the modifying effect of genotype.
\end{abstract}

\section{Key words: Obesity: Type 2 diabetes: CVD: Blood pressure: Catechins}

Diets high in flavonoid-rich foods, including fruits and vegetables, tea, red wine and cocoa, are associated with a range of health benefits which may confer protection against age-related disease, particularly CVD, diabetes and cancer. Flavonoids represent a broad class of plant secondary metabolites most commonly known for their antioxidant activity. Recent evidence, however, indicates that the cardioprotective and metabolic properties of flavonoids may partly be linked to an impact on endothelial function and their modifying effect on the production of vasoconstrictors and vasodilators. For example, improvements in endothelial-dependent vasodilation have been reported after the consumption of cocoa-rich foods such as dark chocolate along with concomitant improvements in blood pressure and insulin sensitivity ${ }^{(1,2)}$. A habitual low intake of cocoa has also been shown to lower blood pressure, the effect being accompanied by a sustained increase in $S$-nitrosoglutathione, which is indicative of improved formation of $\mathrm{NO}$, a potent vasodilator ${ }^{(3)}$. Although understanding on the bioactive components of cocoa mediating these effects is relatively limited, plasma levels of epicatechin have been found to rise after consumption of flavanol-rich cocoa or dark chocolate and this is in concert with improvements in endothelial function $^{(4,5)}$. Ingestion of pure (-)-epicatechin has also been shown to stimulate vasodilation and augment NO status ${ }^{(6)}$.

Green tea provides an alternative dietary source of epicatechin. Other tea catechins, including epigallocatechin gallate (EGCG) which is the major catechin species found in green tea, are also indicated to influence NO production and vasodilation $^{(7)}$. Consistent with this and the link between endothelial dysfunction and hypertension, dietary supplementation with green tea and more recently EGCG has been shown to prevent blood pressure increases in rodents ${ }^{(8-10)}$. In human subjects, however, evidence to date on the effect of tea catechins on blood pressure is inconsistent and limited. Habitual consumption of tea has been associated with a reduced risk of hypertension $^{(11)}$. Moreover, we recently reported a small but significant reduction in diastolic blood pressure in overweight and insulin-resistant subjects after dietary supplementation with $\mathrm{EGCG}^{(12)}$. In contrast, others have observed an acute rise in blood pressure after green tea consumption, with no overall change in blood pressure after short-term ingestion ${ }^{(13)}$.

Abbreviations: COMT, catechol-O-methyltransferase; DGT, decaffeinated green tea extract; EGC, epigallocatechin; EGCG, epigallocatechin gallate. 
Variability in flavonoid metabolism is indicated to affect the health properties of dietary flavonoids ${ }^{(14)}$. O-methylation, which is a major pathway of flavonoid metabolism, is catalysed by the enzyme catechol-O-methyltransferase (COMT). Two distinct forms of COMT have been shown to exist: a soluble protein, which is the predominant form in most tissues, and a membrane-bound form. A common genetic polymorphism ( $G$ to A substitution) has been identified that alters the function of the COMT enzyme. This polymorphism, which produces an amino acid change from Val to Met at codon $108 / 158$ of the resultant soluble/membrane-bound protein, has been shown to reduce the thermostability of the enzyme and is associated with 3- to 4-fold lower enzyme activity ${ }^{(15,16)}$. Given the effect of this polymorphism on thermostability and enzyme activity, it is possible that the COMT Val/Met genotype will influence the rate of catechin metabolism and hence systemic exposure to these compounds. This may in turn influence functional response to dietary catechins.

The purpose of the present study was to determine whether short-term dietary supplementation with green tea catechins lowers blood pressure and improves biomarkers of metabolic function in sedentary and overweight or obese men. The potential modifying effect of the COMT Val/Met genotype was also examined.

\section{Subjects and methods}

\section{Subjects}

Sedentary $(<30$ min of moderate physical activity on most days), non-smoking male subjects, aged 40-69 years, with BMI $\geq 28$ and $\leq 38 \mathrm{~kg} / \mathrm{m}^{2}$ and with no significant history of disease (including anorexia, bulimia or other eating disorders) or current disease were recruited on to the study. Exclusion criteria included: taking prescribed medication; blood pressure $\geq 160 / 100 \mathrm{mmHg}$; upper arm circumference $<24 \mathrm{~cm}$ or $>42 \mathrm{~cm}$; having dairy or wheat allergies; reporting weight change $\pm 10 \%$ during the 6 months before the study; currently following a medically prescribed or slimming diet; taking part in a human study or giving blood during the previous 4 weeks and participation contraindicated, according to the study physician, after review of haematology and clinical chemistry parameters. The study was conducted between October 2007 and June 2008 at the Colworth Laboratory (Sharnbrook, Beds, UK) according to the guidelines laid down in the Declaration of Helsinki. All procedures involving human subjects were approved by the Colworth Science Park Ethics Committee. Written informed consent was obtained from all subjects.

\section{Study design}

This was a single-centre, placebo-controlled, double-blind study with groups matched for age and BMI. The study population was randomly assigned to receive treatments in a complete cross-over design where the allocation was either active (decaffeinated green tea extract; DGT) followed by placebo (lactose) treatment or vice versa. For the majority of subjects, treatment period 1 was early October-early December and treatment period 2 late January-early April. Exceptions to this were two subjects who undertook intervention period 2 between April and June and six subjects where both treatment periods occurred between January and June. Treatment consisted of taking capsules twice daily for 6 weeks, $1 \mathrm{~h}$ before breakfast and $1 \mathrm{~h}$ before the evening meal. Compliance was confirmed by pill count. Between treatments there was a washout period of at least 2 weeks (mean 67.4 (SD 21.5) d). Randomisation was performed by a study-independent statistician. To conceal treatment from the study staff, supplement packs were prepared and labelled by a third-party manufacturer in accordance with the randomisation list. During each of the two intervention phases, subjects visited the study site at week 0 (visits 1 and 3) and after 6 weeks of dietary supplementation (visits 2 and 4). In the week preceding each of the study visits a $24 \mathrm{~h}$ urine sample and dietary information were collected on a convenient weekday. Strenuous exercise and consumption of alcohol, caffeinated products and dark chocolate were restricted for the $24 \mathrm{~h}$ before each study visit and during ambulatory blood pressure monitoring. Subjects were prohibited from eating and drinking, except for water, from 21.00 hours on the day before each study visit until food was provided at the study site the following day. At the study site blood sampling for clinical chemistry measurements was performed, an ambulatory blood pressure monitor fitted and then subjects were provided with a standard breakfast meal (100 g Kellogg's Frosties, $300 \mathrm{ml}$ semi-skimmed milk, $250 \mathrm{ml}$ water (energy $2171 \mathrm{~kJ}$ (519 kcal); $17 \mathrm{~g}$ protein; $99 \mathrm{~g}$ carbohydrate; $6 \mathrm{~g}$ fat)). Subjects remained at the study site for the first $5 \mathrm{~h}$ of the blood pressure measurement period and were then free living, in accordance with protocol restrictions, thereafter. Whilst wearing the blood pressure monitor, subjects were required to record dietary and daily activity information. For study visits 1 and 3, subjects started taking their treatment capsules the evening after completion of the blood pressure-monitoring period. For study site visits 2 and 4 , subjects were requested to take their treatment capsule $1 \mathrm{~h}$ before their scheduled appointment and to continue treatment until blood pressure monitoring was complete. During the intervention phase the consumption of green tea was prohibited.

\section{Dietary supplements}

The placebo treatment was $949 \mathrm{mg}$ lactose per capsule. The active treatment was $530 \mathrm{mg}$ DGT per capsule (Sunphenon 90LB; Taiyo Kagaku Ltd, Yokkaichi, Japan) comprising $>75 \%$ catechins and $<1 \%$ caffeine. The composition of DGT was determined following the procedures described in ISO $14502-2^{(17)}$ and comprised EGCG (40.71\%), EGC (16.27\%), epicatechin (8.74\%), epicatechin gallate $(6.02 \%)$, gallocatechin $(2.02 \%)$, gallocatechin gallate $(1.27 \%)$, catechin $(1 \cdot 16 \%)$, gallic acid $(0.75 \%)$ and catechin gallate $(0.03 \%)$ Opaque, size 00 hydroxypropyl methylcellulose capsules were prepared according to Good Manufacturing Practice by DHP pharma (Crickhowell, Powys, UK), a registered manufacturer, importer and distributor of investigational medicinal products (Marketing Authorisation (Investigational Medicinal 
Product) MA (IMP) 10284). Capsules were weighed and sorted within a weight range of $\pm 5 \%$ and placed within blister packs (twenty-eight capsules per pack). Then three blister packs were placed into each labelled carton. Subjects received two cartons, one at the start of intervention phase 1 and the second at the start of intervention phase 2 .

\section{Anthropometric measurements}

Height $(\mathrm{cm})$ was measured at screening. On each study visit body weight (kg) was recorded and both non-smoking status and alcohol abstinence verified by a Micro CO meter (CareFusion (Micro Medical Ltd), Basingstoke, Hants, UK) and an Alcoscan AL6000 Breathalyser (Sentech, Korea), respectively. All equipment was calibrated before use.

\section{Ambulatory blood pressure monitoring}

Ambulatory blood pressure monitoring was performed using a non-invasive oscillometric device (TM-2430; A\&D Company Ltd, Tokyo, Japan). Blood pressure and heart rate were automatically recorded at defined intervals, which were every $20 \mathrm{~min}$ during $07.00-22.00$ hours and every $30 \mathrm{~min}$ during 22.00-07.00 hours. The median values for $24 \mathrm{~h}$, daytime (09.00-20.59 hours), controlled daytime (measurements over the first $5 \mathrm{~h}$ ) and night-time (01.00-05.59 hours) blood pressure measurements were considered for statistical evaluation. Blood pressure values were calculated using all data collected from the first $24 \mathrm{~h}$, excluding calibration (the first three measurements).

\section{Blood sampling and analysis}

Fasted blood samples were collected and stored at $-80^{\circ} \mathrm{C}$ before analysis. Haematology (leucocyte and erythrocyte count, $\mathrm{Hb}$, packed cell volume, platelets) and clinical chemistry (alanine aminotransferase, aspartate aminotransferase, alkaline phosphatase, $\boldsymbol{\gamma}$-glutamyl-transferase, total bilirubin, albumin, total protein, $\mathrm{Na}, \mathrm{K}$, creatinine, total cholesterol, HDL-cholesterol, LDL-cholesterol, TAG, glucose, insulin) parameters were analysed at Mulhaven Laboratories (Bedford, UK) using MIRA Plus or Immulite (insulin only) instruments.

Plasma concentrations of total EGCG were measured by gradient HPLC with Coularray detection. In brief, $200 \mu \mathrm{l}$ plasma samples were spiked with an internal standard solution (ethyl gallate) at a level of $500 \mathrm{ng} / \mathrm{ml}$, enzymically treated with glucuronidase-sulfatase and extracted three times with ethyl acetate. The combined organic layers were dried under $\mathrm{N}_{2}$, reconstituted in $200 \mu \mathrm{l}$ of stabiliser solution containing EDTA and ascorbic acid, in 2\% acetic acid-10\% acetonitrile and analysed by HPLC (CoulArray ${ }^{\circledR}$; ESA- A Dionex Company, Chelmsford, MA, USA). Chromatographic separation was carried out on a Synergi Polar column ( $250 \times 4.6 \mathrm{~mm}, 3 \mu \mathrm{m}$; Phenomenex, Torrance, CA, USA) with the mobile phases being: $50 \mathrm{~mm}$-phosphate buffer ( $\mathrm{pH} \mathrm{3.4)}$ containing $10 \%$ acetonitrile (A) and 50 mm-phosphate buffer ( $\mathrm{pH} 3.4$ ) containing $50 \%$ acetonitrile (B), respectively. An array of potentials was used for detection, with two channel
( $50 \mathrm{mV}$ for EGCG and mono-methylated EGCG, $300 \mathrm{mV}$ for di- and tri-methylated EGCG) selected for quantification.

Genotype analysis for a non-synonymous SNP in the COMT gene (rs4680) was conducted by direct sequencing. Genomic DNA was extracted from whole blood using an AGOWA magnetic Maxi DNA isolation kit on an automated platform (Hamilton, Reno, NV, USA). Then $50 \mathrm{ng}$ of purified genomic DNA was amplified by PCR in $50 \mu$ of $1 \times$ PCR buffer (ABgene, Cambridge, Cambs, UK), $20 \mathrm{~mm}$-deoxyribonucleotide triphosphate, 2.5 units Thermo-Start ${ }^{\circledR}$ DNA polymerase (Thermo-Scientific, Loughborough, Leicestershire, UK), 25 pmol $5^{\prime}$ primer (GGGCTTCAGTAATTTCAGGG) and $25 \mathrm{pmol}$ $3^{\prime}$ primer (AGCCTCAGACATCTCCAGTCC). Cycling conditions were: thirty cycles of $94^{\circ} \mathrm{C}$ for $15 \mathrm{~s}, 55^{\circ} \mathrm{C}$ for $15 \mathrm{~s}, 72^{\circ} \mathrm{C}$ for $120 \mathrm{~s}$, using a PerkinElmer PCR machine (PerkinElmer, Waltham, MA, USA). The initial denaturing step $\left(94^{\circ} \mathrm{C}\right)$ was extended to $15 \mathrm{~min}$. PCR fragments were purified using a MinElute ${ }^{\circledR}$ 96 UF PCR purification kit (QIAGEN, Hilden Germany). Then $10 \mathrm{ng}$ of each purified PCR fragment were sequenced using an ABI PRISM dGTP BigDye terminator cycle sequencing kit (Applied Biosystems, Carlsbad, CA, USA); unincorporated fluorescent nucleotides were removed using a CleanSeq SPRI magnetic clean up kit (Beckman Coulter Genomics (formerly Agencourt Biosciences), Danvers, MA, USA). Sequencing reactions were separated on an ABI 3730 DNA sequencer and data analysed using the DNASTAR Seqman II software package (Lasergene®; DNASTAR, Inc., Madison, WI, USA).

\section{Urine collection and analysis}

Subjects were asked to discard their first early morning urine (start time) on each collection day, and then collect all their urine, in a beaker containing $200 \mathrm{mg}$ ascorbate, for a period of $24 \mathrm{~h}$, up to and including the urine produced at the start time on the following day. The total sample for the $24 \mathrm{~h}$ was mixed to make a homogeneous solution and the total volume determined by weight. Samples were acidified with $1 \mathrm{M}$-hydrochloric acid before being stored at $-80^{\circ} \mathrm{C}$. Concentrations of EGC and $4^{\prime}$-O-methyl EGC were determined by liquid chromatography-MS (LC-MS). In brief, $50 \mu$ l samples of urine were treated as described above for plasma and the obtained sample extracts injected onto the LC-MS system (Waters Alliance HPLC system coupled to a Waters ZMD mass spectrometer; Waters Corp., Milford, MA, USA). The chromatographic column was Synergi $4 \mathrm{u}$ Polar-RP $80 \mathrm{~A}(150 \times 2 \mathrm{~mm}$, Phenomenex) maintained at $30^{\circ} \mathrm{C}$ and the mobile phase consisted of $0.1 \%$ formic acid in water-acetonitrile. Mass detection for EGC and 4'-O-methyl EGC was carried out in positive electrospray mode recording single ion traces at $m / z 307$ (EGC) and $m / z 321$ (4'-O-methyl EGC) and in negative ion mode for ethyl gallate (internal standard) at $m / z 197$.

\section{Measurement of diet and daily living activities}

Dietary information was collected during periods of urine collection and ambulatory blood pressure monitoring using $24 \mathrm{~h}$ food diaries. Information was analysed using WISP 
analysis software (Tinuviel Software, Llanfechell, Angelsey, UK). Information on daily living activities during ambulatory blood pressure monitoring was recorded in paper diaries.

\section{Statistical analysis}

Taking a two-sided $5 \%$ significance level, the study was calculated to require a minimum of sixty subjects to achieve $85 \%$ power to detect a difference (change from baseline) in blood pressure of $3 \mathrm{mmHg}$ between placebo and active treatments. This was calculated assuming a standard deviation of $7.5 \mathrm{mmHg}$ for both treatments and a correlation of 0.5 for blood pressure between treatments. These assumptions were derived from a pilot study (CC Tumlin, LD Sassano and HAE Hendrickx, unpublished results). Statistical methods were defined before unblinding. Briefly, for primary variables, mean changes (from baseline to the end of the 6-week treatment period) in median $24 \mathrm{~h}$ systolic and median $24 \mathrm{~h}$ diastolic blood pressure for DGT and placebo treatments were compared using a mixed model with treatment as the fixed effect to be tested. A two-sided 5\% significance level was used for each endpoint and a result declared significant if $P<0.05$; no adjustment for multiple testing was made. Analyses were adjusted for the following fixed effect covariates: period of treatment, baseline blood pressure, age at briefing, and baseline BMI. All baselines were calculated within treatment for each subject. Fixed-effect covariates were retained in the model without regard to their degree of significance. Subject number was included as a random effect. Sensitivity analysis was conducted for each outcome variable. Subjects where either of the standardised residuals exceeded an absolute value of 3.5 or where the solution obtained from the random effects (the EBLUP method (empirical best linear unbiased prediction)) had a $P$ value less than 0.005 were removed from the analysis. The treatment effect was only reported for the sensitivity analysis and the outlier removal procedure was only carried out once for each analysis. Similar analyses were performed for the many secondary variables. To assess the modifying effect of COMT genotype on urinary levels of EGC and 4'-O-methyl EGC, four simple models were applied to the logarithms of the analytic values obtained at the end of the intervention period. Firstly, a $2 \mathrm{df}$ ANOVA was used to produce estimated means (with 95\% CI) for each genotype. These were then transformed back to the original $\mathrm{mg} / 24 \mathrm{~h}$ scale for ease of interpretation. Secondly, three separate statistical models corresponding to additive, dominant and recessive genetic models were applied. $P$ values for the COMT genotype term in all four statistical models were produced. All models included covariates for age and BMI. Data are presented as mean values and standard deviations unless stated otherwise.

\section{Results}

There were eighty-three subjects entered on to the study and a total of sixty-four subjects completed both intervention phases. The flow of participants through the phases of the study is shown in Supplementary Fig. 1 (available online at http://www.journals.cambridge.org/bjn). All randomised subjects with at least one valid endpoint were included in the intention-to-treat dataset, which comprised data from seventy-one subjects (placebo $n$ 70; DGT $n$ 67). There were five subjects who were deemed not to have adhered to the requirements of the protocol; these were excluded from the per-protocol dataset (placebo $n$ 65; DGT $n$ 63). Subject exclusion occurred before release of the double-blind procedure. We excluded two subjects due to insufficient blood pressure readings ( $<75 \%$ available for the $24 \mathrm{~h}$ period) and three subjects were excluded because their body weight fluctuated by more than $5 \%$ within at least one of the intervention phases.

To monitor compliance, subjects were required to return all packaging and unused capsules. Each subject received eightyfour placebo and eighty-four DGT capsules. For subjects ( $n$ 64) that completed the full trial an average of 1.36 (SD 1.92) placebo capsules and 1.14 (SD 2.19) DGT capsules were returned per subject, indicating that in general $>98 \%$ of doses were taken. A total of seventy-nine adverse events (forty-two placebo group; thirty-seven DGT group) were reported during the course of the study (see Supplementary Table 1; available online at http://www.journals.cambridge. org/bjn). These were all categorised as mild and no difference in type or quantity was detected between the two groups.

Baseline characteristics for the placebo and DGT groups are shown in Table 1 . No significant differences were detected between the two groups. It was noted that $64 \%$ of the placebo group and $73 \%$ of the DGT group were obese (BMI $\geq$ $30 \mathrm{~kg} / \mathrm{m}^{2}$ ). The presence of mild hypertension (systolic blood pressure $\geq 140$ but $<160 \mathrm{mmHg}$ and/or diastolic blood pressure $\geq 90$ but $<100 \mathrm{mmHg}: 8.6$ and $10 \cdot 4 \%$ for placebo and DGT groups, respectively) and pre-hypertension (systolic blood pressure 130-139 $\mathrm{mmHg}$ and/or diastolic blood pressure 85-89 mmHg: 28.6 and $28.4 \%$ for placebo and DGT groups, respectively) was detected in a subset of subjects.

Plasma concentrations of EGCG, the major catechin species in the DGT, were undetectable at baseline and remained undetectable after dietary supplementation in the placebo group but increased to an average level of $98 \mathrm{ng} / \mathrm{ml}$ in the DGT group (Table 2). Urinary excretion over $24 \mathrm{~h}$ of EGC and $4^{\prime}$-O-methyl EGC was similar for the two groups at baseline. Post-intervention, the excretion of EGC and $4^{\prime}-O$-methyl EGC remained unchanged in the placebo group but increased in the DGT group by 28 -fold and 34-fold, respectively (Table 2).

Using the intention-to-treat dataset, change from baseline at week 6 was compared between placebo and DGT groups for all outcome measures. No effect of treatment on any of the ambulatory blood pressure-monitoring parameters was detected (Table 3). A small effect $(P=0.02)$ on LDL-cholesterol was observed, with levels reducing in the DGT group after treatment and increasing in the placebo group. However, after removal of a single outlier the significance level decreased to $P=0 \cdot 04$, most probably indicating type 1 error given the multiple testing burden present within the analysis of the secondary variables. DGT treatment had no significant effect on any of the other biomarkers of metabolic function (Table 3). Similar results were obtained from the per-protocol analysis (data not shown). 
Table 1. Baseline characteristics of the intention-to-treat dataset (Mean values and standard deviations)

\begin{tabular}{|c|c|c|c|c|c|c|c|}
\hline \multirow[b]{2}{*}{ Variable } & \multicolumn{3}{|c|}{ Placebo } & \multicolumn{3}{|c|}{ DGT } & \multirow[b]{2}{*}{$P^{*}$} \\
\hline & $n$ & Mean & SD & $n$ & Mean & SD & \\
\hline Age (years) & 70 & $49 \cdot 4$ & 5.6 & 67 & 49.5 & 5.6 & 0.95 \\
\hline BMI $\left(\mathrm{kg} / \mathrm{m}^{2}\right)$ & 70 & 31.4 & 2.6 & 67 & $31 \cdot 7$ & $2 \cdot 7$ & 0.53 \\
\hline Body weight $(\mathrm{kg})$ & 70 & $100 \cdot 2$ & $11 \cdot 1$ & 67 & $101 \cdot 3$ & 11.4 & 0.57 \\
\hline \multicolumn{8}{|l|}{ Blood pressure $(\mathrm{mmHg})$} \\
\hline \multicolumn{8}{|l|}{ SBP } \\
\hline $24 \mathrm{~h}$ & 70 & $127 \cdot 7$ & 8.3 & 67 & $127 \cdot 1$ & 8.7 & 0.69 \\
\hline Controlled daytime & 70 & $130 \cdot 0$ & $9 \cdot 4$ & 67 & 130.5 & 9.2 & 0.73 \\
\hline Daytime & 70 & $134 \cdot 3$ & 8.9 & 67 & 133.4 & 9.2 & 0.55 \\
\hline Night-time & 70 & $110 \cdot 8$ & 9.5 & 67 & $112 \cdot 3$ & $10 \cdot 2$ & 0.38 \\
\hline \multicolumn{8}{|l|}{ DBP } \\
\hline $24 \mathrm{~h}$ & 70 & 79.5 & 5.9 & 67 & 79.1 & 6.1 & 0.64 \\
\hline Controlled daytime & 70 & 82.5 & $7 \cdot 0$ & 67 & $82 \cdot 7$ & $6 \cdot 8$ & 0.86 \\
\hline Daytime & 70 & $83 \cdot 7$ & 6.4 & 67 & 83.5 & $6 \cdot 7$ & 0.89 \\
\hline Night-time & 70 & $69 \cdot 1$ & $7 \cdot 6$ & 67 & $69 \cdot 2$ & $7 \cdot 1$ & 0.98 \\
\hline Heart rate, $24 \mathrm{~h}$ (beats $/ \mathrm{min}$ ) & 70 & $68 \cdot 8$ & $8 \cdot 7$ & 67 & $67 \cdot 1$ & $8 \cdot 2$ & 0.24 \\
\hline \multicolumn{8}{|l|}{ Cholesterol $(\mathrm{mmol} / \mathrm{l})$} \\
\hline Total & 70 & 5.4 & 1.0 & 67 & $5 \cdot 3$ & 1.1 & 0.56 \\
\hline $\mathrm{HDL}$ & 70 & 1.2 & 0.3 & 67 & 1.2 & 0.3 & 0.75 \\
\hline LDL & 70 & 3.4 & 0.8 & 67 & 3.3 & 0.9 & 0.55 \\
\hline TAG (mmol/l) & 70 & 1.8 & 1.0 & 67 & $1 \cdot 8$ & 0.9 & 0.94 \\
\hline Glucose $(\mathrm{mmol} / \mathrm{l})$ & 70 & 6.0 & 0.6 & 67 & 5.9 & 0.5 & 0.65 \\
\hline Insulin (pmol//) & 69 & $86 \cdot 81$ & 44.45 & 67 & 84.03 & $43 \cdot 75$ & 0.69 \\
\hline \multicolumn{8}{|l|}{ Dietary values (g/d) } \\
\hline Alcohol & 66 & 0.00 & 0.00 & 62 & 0.01 & 0.08 & 0.30 \\
\hline Carbohydrate & 66 & 309.9 & $101 \cdot 0$ & 62 & $298 \cdot 2$ & $105 \cdot 5$ & 0.52 \\
\hline Fat & 66 & $106 \cdot 8$ & 39.9 & 62 & $97 \cdot 7$ & $31 \cdot 7$ & 0.16 \\
\hline Protein & 66 & $102 \cdot 0$ & $32 \cdot 7$ & 62 & $97 \cdot 1$ & $27 \cdot 6$ & 0.36 \\
\hline Estimated energy intake & & & & & & & 0.23 \\
\hline $\mathrm{kJ} / \mathrm{d}$ & 66 & 10605 & 2927 & 62 & 10011 & 2596 & \\
\hline $\mathrm{kcal} / \mathrm{d}$ & 66 & 2533 & 699 & 62 & 2391 & 620 & \\
\hline \multicolumn{8}{|l|}{ Catechin levels (ng/ml) } \\
\hline Urinary EGC & 70 & $288 \cdot 8$ & $188 \cdot 1$ & 67 & $310 \cdot 3$ & $228 \cdot 9$ & 0.55 \\
\hline Urinary $4^{\prime}-O$-methyl EGC & 70 & $104 \cdot 3$ & $89 \cdot 1$ & 67 & $106 \cdot 1$ & $96 \cdot 3$ & 0.91 \\
\hline
\end{tabular}

DGT, decaffeinated green tea extract; SBP, systolic blood pressure; DBP, diastolic blood pressure; EGC, epigallocatechin. * Differences between groups ( $t$ test).

As body weight is known to influence blood pressure, change in body weight was investigated as a potential confounding factor. Although no overall treatment effect was detected, mixed-model analysis did reveal a significant period $\times$ treatment interaction $(P<0 \cdot 05)$, indicating a possible seasonal effect. Exploratory analysis was therefore performed using a subset of the intention-to-treat dataset which comprised those subjects with all four data points available for calculating weight change who had completed the study protocol between October and April. A significant treatment effect $(P=0.025)$ was detected during intervention period 1 , with subjects in the placebo group gaining on average 0.53 (SD 1.9) $\mathrm{kg}$ of body weight whilst those in the DGT group showed on average a 0.64 (SD 2.2) $\mathrm{kg}$ reduction in body

Table 2. Effect of dietary supplementation on plasma and urinary catechin levels in the intention-to-treat dataset

\begin{tabular}{|c|c|c|c|c|c|c|c|c|c|c|c|c|}
\hline \multirow[b]{2}{*}{$\begin{array}{l}\text { Variable } \\
\text { (ng/ml) }\end{array}$} & \multicolumn{6}{|c|}{ Placebo } & \multicolumn{6}{|c|}{ DGT } \\
\hline & $n$ & Median & $\begin{array}{l}\text { Lower } \\
\text { quartile }\end{array}$ & $\begin{array}{c}\text { Upper } \\
\text { quartile }\end{array}$ & Minimum & Maximum & $n$ & Median & $\begin{array}{l}\text { Lower } \\
\text { quartile }\end{array}$ & $\begin{array}{l}\text { Upper } \\
\text { quartile }\end{array}$ & Minimum & Maximum \\
\hline \multicolumn{13}{|l|}{ EGCG } \\
\hline Week 0 & 69 & 0 & 0 & 0 & 0 & 29 & 65 & 0 & 0 & 0 & 0 & 30 \\
\hline Week 6 & 68 & 0 & 0 & 0 & 0 & 165 & 65 & 98 & 43 & 150 & 0 & 755 \\
\hline \multicolumn{13}{|l|}{ EGC } \\
\hline Week 0 & 70 & 243 & 171 & 356 & 27 & 997 & 68 & 259 & 177 & 346 & 30 & 1017 \\
\hline Week 6 & 69 & 241 & 166 & 346 & 63 & 1137 & 66 & 6885 & 5340 & 10296 & 319 & 49845 \\
\hline \multicolumn{13}{|l|}{$\begin{array}{l}4^{\prime}-O \text {-methyl } \\
\text { EGC }\end{array}$} \\
\hline Week 0 & 70 & 74 & 47 & 130 & 7 & 440 & 68 & 68 & 46 & 135 & 12 & 497 \\
\hline Week 6 & 69 & 74 & 32 & 135 & 6 & 449 & 66 & 2517 & 1588 & 3756 & 82 & 23776 \\
\hline
\end{tabular}

DGT, decaffeinated green tea extract; EGCG, epigallocatechin gallate; EGC, epigallocatechin. 
Table 3. Effect of dietary supplementation on outcome variables in the intention-to-treat datasetł (Mean values and standard deviations)

\begin{tabular}{|c|c|c|c|c|c|c|c|}
\hline \multirow[b]{2}{*}{ Variable } & \multicolumn{3}{|c|}{ Placebo } & \multicolumn{3}{|c|}{ DGT } & \multirow[b]{2}{*}{$P^{*}$} \\
\hline & $n$ & Mean & SD & $n$ & Mean & SD & \\
\hline \multicolumn{8}{|l|}{ Blood pressure $(\mathrm{mmHg})$} \\
\hline \multicolumn{8}{|l|}{ SBP } \\
\hline $24 \mathrm{~h}$ & 69 & 0.24 & 5.63 & 66 & 0.54 & $5 \cdot 61$ & 0.62 \\
\hline Controlled daytime & 69 & -0.07 & $9 \cdot 24$ & 66 & 0.23 & $7 \cdot 64$ & 0.78 \\
\hline Daytime & 69 & -0.16 & 5.91 & 66 & 0.68 & 6.34 & 0.66 \\
\hline Night-time & 68 & -0.38 & $8 \cdot 36$ & 66 & -2.02 & 8.94 & 0.38 \\
\hline \multicolumn{8}{|l|}{ DBP } \\
\hline $24 \mathrm{~h}$ & 69 & 0.01 & 4.61 & 66 & 0.39 & $3 \cdot 70$ & 0.91 \\
\hline Controlled daytime & 69 & -0.39 & 5.02 & 66 & 0.03 & 4.87 & 0.84 \\
\hline Daytime & 69 & 0.06 & 4.85 & 66 & 0.48 & 4.19 & 0.40 \\
\hline Night-time & 68 & -1.32 & 6.65 & 66 & -1.33 & $6 \cdot 67$ & 0.80 \\
\hline Heart rate, $24 \mathrm{~h}$ (beats $/ \mathrm{min}$ ) & 69 & 0.98 & 4.35 & 66 & 2.59 & 3.86 & 0.086 \\
\hline \multicolumn{8}{|l|}{ Cholesterol (mmol/l) } \\
\hline Total & 66 & 0.07 & 0.59 & 63 & -0.04 & 0.46 & 0.15 \\
\hline $\mathrm{HDL}$ & 66 & -0.01 & 0.18 & 63 & -0.01 & 0.14 & 0.96 \\
\hline LDL & 66 & 0.08 & 0.50 & 63 & -0.10 & 0.43 & 0.023 \\
\hline TAG $(\mathrm{mmol} / \mathrm{l})$ & 66 & 0.04 & 0.67 & 63 & 0.13 & 0.55 & 0.42 \\
\hline Glucose (mmol/l) & 66 & -0.14 & 0.42 & 63 & -0.19 & 0.48 & 0.45 \\
\hline Insulin (pmol/l) & 65 & 2.29 & 30.97 & 63 & 0.63 & $30 \cdot 14$ & 0.66 \\
\hline Body-weight change $(\mathrm{kg})$ & 69 & -0.038 & 1.88 & 66 & -0.327 & 1.89 & $0.037 \dagger$ \\
\hline
\end{tabular}

weight. In contrast, during intervention period 2 body-weight change was similar for both placebo and DGT groups, reducing by 0.55 (SD 2.0$) \mathrm{kg}$ and 0.32 (SD 1.4) $\mathrm{kg}$, respectively (Fig. 1). Over intervention periods 1 and 2 the estimated energy intake of the study population changed by 716 (SD $3035)$ and -502 (SD 3366) $\mathrm{kJ} / \mathrm{d}(171$ (SD 725) and -120 (SD $804) \mathrm{kcal} / \mathrm{d})$, respectively. During intervention period 1 the increase in estimated energy intake was similar $(P=0.37)$ for the placebo and DGT groups. However, during intervention period 2 a group difference was detected $(P=0 \cdot 022)$, with estimated energy intake decreasing in the placebo group $(-1478$ (SD 3538) $\mathrm{kJ} / \mathrm{d}$; $-353(\mathrm{SD} 845) \mathrm{kcal} / \mathrm{d}$ ) but increasing in the DGT group (636 (SD 2818) kJ/d; 152 (SD 673) kcal/d).

The modifying effect of the COMT Val/Met genotype was examined, as this common genetic polymorphism has been found to alter the stability and activity of the resultant enzyme and may influence the health properties of tea catechins. Mean urinary levels of EGC and 4'-O-methyl EGC were similar for the AA and AG genotype groups but markedly lower for the GG genotype group (Table 4). Exploratory modelling indicated that a recessive genetic model for the $G$ allele fitted the data well. No consistent and robust genotype $\times$ treatment interactions were detected for body-weight change or any of the other outcome variables (data not shown).

\section{Discussion}

The effect of regular consumption of green tea catechins was assessed in a population of sedentary, middle-aged men who were overweight or obese. Subjects consumed $530 \mathrm{mg}$ of DGT, twice daily. This provided an intake of $800 \mathrm{mg}$ total catechins per $\mathrm{d}$ and is comparable with drinking about six to eight cups of moderate-strength green tea ${ }^{(18)}$. A marked increase in the plasma concentration of EGCG and urinary concentration of EGC and 4'-O-methyl EGC was detected after DGT treatment, indicating absorption of catechins. Although DGT treatment had no observable effect on blood pressure, heart rate,

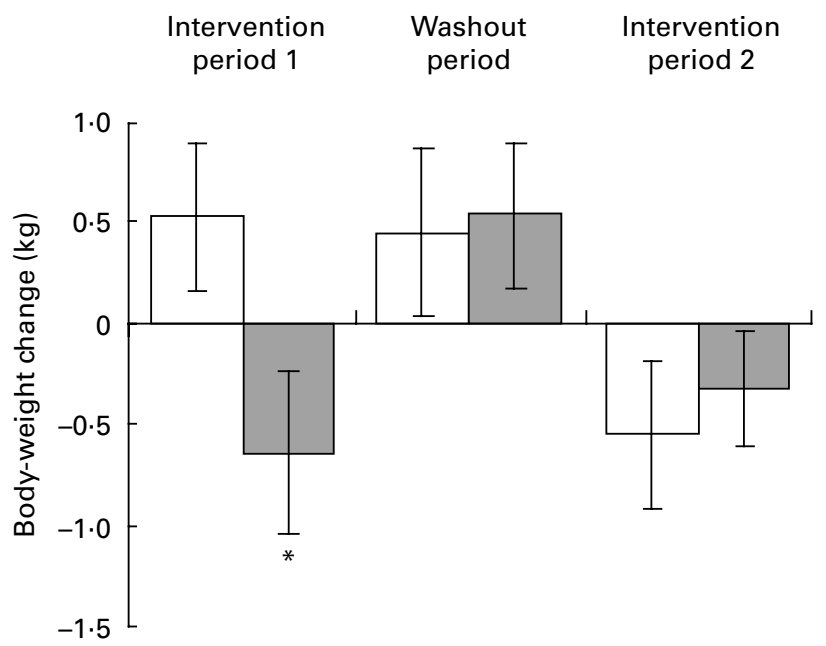

Fig. 1. Effect of dietary supplementation on body-weight change. Data are shown for a refined intention-to-treat dataset which comprised those subjects in the placebo $(\square)$ and decaffeinated green tea extract $(\square)$ groups with full data for body-weight change and who completed the study protocol between October and April ( $n$ 56). Values are means, with standard errors represented by vertical bars. * Mean value was significantly different from that the placebo group $(P=0.025)$. There was a period $\times$ treatment interaction $(P=0.037)$. 
Table 4. Modifying effect of catechol-O-methyltransferase genotype on urinary levels of epigallocatechin (EGC) and 4'-O-methyl EGC †

\begin{tabular}{|c|c|c|c|c|c|c|c|c|}
\hline Variable (mg) & Genotype & LSMean & $\begin{array}{c}\text { Lower } \\
95 \% \text { CL }\end{array}$ & $\begin{array}{c}\text { Upper } \\
95 \% \text { CL }\end{array}$ & Model-free $^{*}$ & $\begin{array}{l}\text { Additive } \\
\text { model }^{*}\end{array}$ & $\begin{array}{c}\text { Dominant } \\
\text { model }^{\star}\end{array}$ & $\begin{array}{l}\text { Recessive } \\
\text { model }^{*}\end{array}$ \\
\hline \multirow[t]{3}{*}{ EGC } & AA & $15 \cdot 38$ & $10 \cdot 41$ & $22 \cdot 72$ & 0.013 & 0.0081 & 0.24 & 0.0035 \\
\hline & $A G$ & 13.85 & 11.21 & $17 \cdot 13$ & & & & \\
\hline & GG & 8.03 & $5 \cdot 82$ & $11 \cdot 10$ & & & & \\
\hline \multirow[t]{3}{*}{ 4'-O-methyl EGC } & AA & $5 \cdot 16$ & $3 \cdot 31$ & 8.05 & 0.029 & 0.021 & 0.37 & 0.0075 \\
\hline & $A G$ & 4.89 & $3 \cdot 84$ & $6 \cdot 22$ & & & & \\
\hline & GG & 2.75 & 1.90 & 3.97 & & & & \\
\hline
\end{tabular}

LSMean, least-squares mean, CL, confidence limit.

${ }^{*} P$ value for different genetic models: ANOVA with age and $B M I$ included as covariates; model-free 2 df non-coded; additive $1 \mathrm{df}$ coded $A A=0, A G=1$, GG $=2 ;$ dominant $1 \mathrm{df}$ coded $A A=0, A G=1, G G=1$; recessive $1 \mathrm{df}$ coded $A A=0, A G=0, G G=1$.

† Data are shown according to genotype for the decaffeinated green tea group at week 6 : AA, $n 11 ; \mathrm{AG}, n 35 ; \mathrm{GG}, n 15$.

or biomarkers of metabolic function, a period $\times$ treatment interaction was noted for body-weight change. Additionally, the COMT Val/Met genotype was found to have a modifying effect on catechin excretion.

Data from epidemiological studies suggest that the habitual consumption of green tea may be beneficial for the prevention of $\mathrm{CVD}^{(19)}$, with some evidence indicating a protective effect against hypertension ${ }^{(11,20,21)}$. In several rodent models dietary supplementation with green tea or green tea catechins is indicated to improve blood pressure, although the findings from studies are not entirely consistent ${ }^{(8-10,22-25)}$. In the present human study, no effect on blood pressure was detected after 6 weeks of dietary supplementation with DGT. A small number of other short-term human intervention studies have investigated the antihypertensive properties of green tea at daily catechin intakes of between 400 and $800 \mathrm{mg}$, although none has applied ambulatory blood pressure monitoring. Previously, we observed a small but significant reduction in diastolic blood pressure of about $3 \mathrm{mmHg}$ in overweight and insulin-resistant subjects after dietary supplementation with EGCG for 8 weeks ${ }^{(12)}$. Fukino et al. similarly found a borderline reduction in diastolic blood pressure of about 9 $\mathrm{mmHg}$ in subjects with elevated fasting glucose after consuming catechins for 2 months ${ }^{(26)}$. Conversely, a reduction in systolic blood pressure of about $7 \mathrm{mmHg}$ was demonstrated in centrally obese subjects after 12 weeks of regular catechin consumption but only in subjects with an initial systolic blood pressure of $\geq 130 \mathrm{mmHg}^{(27)}$. In contrast, and consistent with the data here, no effect on blood pressure was noted by Frank et al. in a population of healthy male subjects after 3 weeks of catechin consumption ${ }^{(28)}$. The discrepancy in findings between these studies may be attributable to a number of factors, including differences in the type of product given, the dose and composition of catechins, the way the test product was administered, the length of the intervention period and the nature of the study population.

The endothelium plays an important role in cardiovascular homeostasis. Impaired endothelial function, characterised by reduced NO bioavailability, is associated with atherosclerosis and other cardiovascular disorders, including hypertension. Indeed, deficiency of $\mathrm{NO}$ is noted in several forms of experimental hypertension, although the importance of this as a principal mediating event does differ between models ${ }^{(29)}$. While studies to date investigating the blood pressure-lowering effects of green tea catechins have yielded mixed results, there is accumulating evidence to indicate that green tea and specific tea catechins have a positive effect on endothelial dysfunction $^{(7)}$. For example, EGCG stimulates $\mathrm{NO}$ production in vitro ${ }^{(30,31)}$ and in rodents induces dosedependent vasodilation in pre-contracted aortic rings ${ }^{(30)}$ and mesenteric vascular beds ${ }^{(10)}$. In human subjects, improvements in endothelial-dependent vasorelaxation have also been observed after the acute consumption of EGCG and green tea ${ }^{(32-34)}$. As neither endothelial function nor NO bioavailability was directly investigated in the present study, it is not possible to ascertain whether these parameters were similarly improved here. Indeed, the present study results may reflect the need for a sustained effect on endothelial function before vascular tone and blood pressure are significantly altered rather than a lack of effect on endothelial function per se. It is also important to recognise that the regulation of blood pressure is multi-factorial and that dietary improvement of endothelial function alone may not necessarily confer a blood pressure-lowering benefit.

Data from in vitro and animal studies indicate that the consumption of green tea may improve insulin resistance and confer metabolic benefits. For example, extracts of green tea have been found to ameliorate the development of insulin resistance in high-fat-fed and high-fructose-fed rodent models and increase insulin sensitivity in male SpragueDawley rats ${ }^{(25,35,36)}$. Dietary supplementation with EGCG has also been demonstrated to improve glucose tolerance and insulin sensitivity in rodents ${ }^{(10,37)}$. In cell-culture models, extracts of green tea are indicated to act as insulin mimetics, with EGCG identified as the principal bioactive species $^{(38,39)}$. EGCG is also known to inhibit activation of Iк kinase ${ }^{(40-42)}$, a Ser/Thr kinase implicated in the pathogenesis of insulin resistance $^{(43)}$ and to modulate the signalling pathway of the Forkhead box class $\mathrm{O}$ transcription factor, which is involved in the regulation of hepatic gluconeogenesis ${ }^{(44)}$. In contrast, the results of several human studies evaluating the effect of regular consumption of green tea catechins on markers of insulin resistance have been largely disappointing ${ }^{(12,26,45,46)}$. Similarly, in the present study no change in fasting serum insulin levels was observed after DGT treatment, suggesting no effect on hepatic insulin resistance. However, it is possible that the metabolic benefits of green tea are principally mediated by postprandial improvements in insulin action, 
which were not evaluated here. Indeed, in a recent study conducted in healthy young men, acute ingestion of a green tea extract improved postprandial insulin sensitivity and glucose tolerance but did not affect fasting plasma glucose or serum insulin concentrations ${ }^{(47)}$

Green tea has been suggested to have anti-obesity effects. In a recent meta-analysis the consumption of green tea catechins was found to have a small positive effect on both weight loss and weight maintenance ${ }^{(48)}$. Consistent with this, here we observed an effect of DGT treatment on bodyweight change during intervention period 1. Despite a similar rise in estimated energy intake for both groups during this period, the body weight of subjects increased in the placebo group but decreased in the DGT group, suggesting a protective effect of green tea catechins on weight gain. Similar to our findings, dietary supplementation with EGCG or a mixture of tea catechins has been shown to reduce high-fat diet-induced body-weight gain in rodents. One implicated mechanism is the stimulation of fat oxidation, possibly via the activation of the LKB1/AMP-activated protein kinase pathway $^{(49)}$ or through the modulation of COMT activity ${ }^{(48)}$. Indeed, as well as being a substrate for COMT, catechins are known to inhibit the activity of this enzyme ${ }^{(50)}$ which is involved in the degradation of noradrenaline, a key hormonal regulator of fat utilisation. The anti-obesity effects of catechins may also be mediated by the modulation of adipogenesis, fat synthesis and nutrient absorption ${ }^{(51)}$. Although both groups lost body weight during intervention period 2, for DGTtreated subjects this was despite an increase in estimated energy intake and is consistent with the protective effect of green tea catechins on weight gain observed during intervention period 1 .

A further observation from the present study was that the COMT Val/Met genotype was found to influence urinary accumulation over $24 \mathrm{~h}$ of EGC and $4^{\prime}$-O-methyl EGC. Lower mean concentrations were noted for individuals homozygous for the high-activity G-allele compared with individuals carrying at least one copy of the low-activity A-allele. Although not investigated here, this may reflect genotype differences in metabolic flux, with conversion of tea catechins to downstream metabolites occurring most rapidly in individuals homozygous for the G-allele. The health effects of green tea are largely attributed to the catechin family of tea flavonoids. As such, factors which modulate the systemic availability of catechins and/or their downstream metabolites could have a significant impact on the health properties associated with tea consumption. For example, an interaction between tea intake, COMT Val/Met genotype and breast cancer risk has previously been described in Asian-American women ${ }^{(52)}$. The protective effect of tea consumption on breast cancer risk was mainly confined to women carrying at least one copy of the low-activity allele. This supports a recessive genetic model for the G-allele and is consistent with the effect of genotype on urinary catechin accumulation observed here. The prevalence of the COMT Val/Met genotype is known to differ between ethnic groups and due to this has been suggested as a factor which may partly explain the moderating effect of ethnicity on the anti-obesity properties of green tea in
Asian and Caucasian populations ${ }^{(48)}$. Although in the present study we did not detect a genotype $\times$ treatment interaction for body-weight change, because of the small sample size this may reflect a limited power to detect such interactions rather than a true null result. Further studies with larger sample sizes are needed to evaluate this possibility.

The present study has a number of limitations that need to be acknowledged. The sample size was relatively small and the present study population was largely normotensive. Given the positive linear relationship between baseline blood pressure and the efficacy of antihypertensives to reduce blood pressure ${ }^{(53)}$, it is possible that a change in blood pressure in response to the intervention may not have been detected if the effect size was small. Furthermore, the duration of our intervention was relatively short and a longer period of dietary supplementation may be required for the blood pressure-lowering effect of green tea to manifest. The present study was of cross-over design, an inherent weakness of which is the potential for treatment order effects. Although an effect of DGT treatment on body-weight change was detected during exploratory post hoc analysis, this was only during the first treatment period. As such, the strength of evidence is more limited and the present results do need to be interpreted with appropriate caution. Given the timing of the present study, it is possible that seasonal differences in food intake may have influenced body-weight change. Indeed, estimated energy intake was noted to rise over intervention period 1 but decrease over intervention period 2. This difference may explain the inconsistency in our findings on body-weight change and raises the prospect that the consumption of green tea catechins may usefully contribute to the prevention of weight gain rather than promoting weight loss per se. This possibility should be investigated in future studies. Furthermore, it needs to be acknowledged that a seasonal shift in food intake during the study could have more broadly influenced the present results, including the effect of DGT on blood pressure. However, we found no evidence for an impact of treatment period on the treatment effect for the primary or any of the other secondary outcome variables.

In summary, the daily consumption of DGT for 6 weeks was well tolerated by sedentary and overweight or obese male subjects. Although DGT treatment did not affect blood pressure, heart rate or biomarkers of metabolic function, a period $\times$ treatment interaction for body-weight change was detected, suggesting a protective effect of green tea catechins on weight gain during periods of positive energy imbalance. COMT genotype was also shown to influence urinary catechin accumulation. Additional studies are required to confirm these findings and to further explore the modifying effect of genotype.

\section{Acknowledgements}

We acknowledge Jacqueline Coverly, Helena Meadows, Janice Stocks, Emmanuel Mubwandarikwa, Ian Smith and Carl Jarman for technical support. 
A. L. B. was responsible for the study concept and preparation of the manuscript. J. L. and C. H. were responsible for conducting the study and for data collection and management. A. E. M. was responsible for genotype analysis. B. N. was responsible for catechin analysis. T. D. was responsible for data analysis. All authors contributed to the study design and data interpretation. T. D., J.L., A. E. M. and B. N. critically reviewed the manuscript.

The authors are all employed by Unilever Research \& Development, which is a division of Unilever plc, a company which has a significant commercial interest in tea. Unilever plc provided all funding for the study.

\section{References}

1. Grassi D, Necozione S, Lippi C, et al. (2005) Flavanol-rich dark chocolate decreases blood pressure, improves endothelium-dependent vasorelaxation, and ameliorates insulin sensitivity in patients with essential hypertension. Hypertension 46, 890.

2. Grassi D, Desideri G, Necozione S, et al. (2008) Blood pressure is reduced and insulin sensitivity increased in glucoseintolerant, hypertensive subjects after 15 days of consuming high-polyphenol dark chocolate. J Nutr 138, 1671-1676.

3. Taubert D, Roesen R, Lehmann C, et al. (2007) Effects of low habitual cocoa intake on blood pressure and bioactive nitric oxide - a randomized controlled trial. J Am Med Assoc 298 , 49-60.

4. Engler MB, Engler MM, Chen CY, et al. (2004) Flavonoid-rich dark chocolate improves endothelial function and increases plasma epicatechin concentrations in healthy adults. $J \mathrm{Am}$ Coll Nutr 23, 197-204.

5. Schroeter H, Heiss C, Balzer J, et al. (2006) (-)-Epicatechin mediates beneficial effects of flavanol-rich cocoa on vascular function in humans. Proc Nat Acad Sci 103, 1024-1029.

6. Loke WM, Hodgson JM, Proudfoot JM, et al. (2008) Pure dietary flavonoids quercetin and (-)-epicatechin augment nitric oxide products and reduce endothelin-1 acutely in healthy men. Am J Clin Nutr 88, 1018-1025.

7. Moore RJ, Jackson KG \& Minihane AM (2009) Green tea (Camellia sinensis) catechins and vascular function. $\mathrm{Br} J$ Nutr 102, 1790-1802.

8. Henry JP \& Stephenslarson P (1984) Reduction of chronic psychosocial hypertension in mice by decaffeinated tea. Hypertension 6, 437-444.

9. Negishi H, Xu JW, Ikeda K, et al. (2004) Black and green tea polyphenols attenuate blood pressure increases in strokeprone spontaneously hypertensive rats. J Nutr 134, 38-42.

10. Potenza MA, Marasciulo FL, Tarquinio M, et al. (2007) EGCG, a green tea polyphenol, improves endothelial function and insulin sensitivity, reduces blood pressure, and protects against myocardial I/R injury in SHR. Am J Physiol Endocrinol Metab 292, E1378-E1387.

11. Yang YC, Lu FH, Wu JS, et al. (2004) The protective effect of habitual tea consumption on hypertension. Arch Intern Med 164, 1534-1540.

12. Brown AL, Lane J, Coverly J, et al. (2009) Effects of dietary supplementation with the green tea polyphenol epigallocatechin-3-gallate on insulin resistance and associated metabolic risk factors: randomized controlled trial. $\mathrm{Br} J \mathrm{Nutr}$ 101, 886-894.

13. Hodgson JM, Puddey IB, Burke V, et al. (1999) Effects on blood pressure of drinking green and black tea. J Hypertens 117, 457-463.
14. Hodgson JM, Puddey IB, Burke V, et al. (2006) Is reversal of endothelial dysfunction by tea related to flavonoid metabolism? Br J Nutr 95, 14-17.

15. Dawling S, Roodi N, Mernaugh RL, et al. (2001) Catechol-Omethyltransferase (COMT)-mediated metabolism of catechol estrogens: comparison of wild-type and variant COMT isoforms. Cancer Res 61, 6716-6722.

16. Syvanen AC, Tilgmann C, Rinne J, et al. (1997) Genetic polymorphism of catechol-O-methyltransferase (COMT): correlation of genotype with individual variation of S-COMT activity and comparison of the allele frequencies in the normal population and Parkinsonian patients in Finland. Pharmacogenetics 7, 65-71.

17. International Organization for Standardization (2005) ISO 14502-2: 2005. Determination of Substances Characteristic of Green and Black Tea - Part 2: Content of Catechins in Green Tea - Method Using High-Performance Liquid Chromatography. Geneva: ISO.

18. Khokhar S \& Magnusdottir SGM (2002) Total phenol, catechin, and caffeine contents of teas commonly consumed in the United Kingdom. J Agric Food Chem 50, 565-570.

19. Kuriyama S (2008) The relation between green tea consumption and cardiovascular disease as evidenced by epidemiological studies. J Nutr 138, 1548-1553.

20. Hodgson JM, Devine A, Puddey IB, et al. (2003) Tea intake is inversely related to blood pressure in older women. J Nutr 133, 2883-2886.

21. Stensvold I, Tverdal A, Solvoll K, et al. (1992) Tea consumption - relationship to cholesterol, blood-pressure, and coronary and total mortality. Prev Med 21, 546-553.

22. Abe Y, Umemura S, Sugimoto K, et al. (1995) Effect of green tea rich in $\gamma$-aminobutyric-acid on blood-pressure of Dahl salt-sensitive rats. Am J Hypertens 8, 74-79.

23. Antonello M, Montemurro D, Bolognesi M, et al. (2006) Arterial benefits of green tea extract: anti-hypertension and prevention of cardiovascular damage and endothelial dysfunction in angiotensin II-dependent hypertension. Hypertension 48, 765-766.

24. Ikeda M, Suzuki C, Umegaki K, et al. (2007) Preventive effects of green tea catechins on spontaneous stroke in rats. Med Sci Monit 13, BR40-BR45.

25. Wu LY, Juan CC, Hwang LS, et al. (2004) Green tea supplementation ameliorates insulin resistance and increases glucose transporter IV content in a fructose-fed rat model. Eur J Nutr 43, 116-124.

26. Fukino Y, Ikeda A, Maruyama K, et al. (2007) Randomized controlled trial for an effect of green tea-extract powder supplementation on glucose abnormalities. Eur J Clin Nutr 62, 953-960.

27. Nagao T, Hase T \& Tokimitsu I (2007) A green tea extract high in catechins reduces body fat and cardiovascular risks in humans. Obesity 15, 1473-1483.

28. Frank J, George TW, Lodge JK, et al. (2009) Daily consumption of an aqueous green tea extract supplement does not impair liver function or alter cardiovascular disease risk biomarkers in healthy men. $J$ Nutr 139, 58-62.

29. Torok J (2008) Participation of nitric oxide in different models of experimental hypertension. Physiol Res 57, $813-825$.

30. Lorenz M, Wessler S, Follmann E, et al. (2004) A constituent of green tea, epigallocatechin-3-gallate, activates endothelial nitric oxide synthase by a phosphatidylinositol-3-OH-kinase-, cAMP-dependent protein kinase-, and Akt-dependent pathway and leads to endothelial-dependent vasorelaxation. J Biol Chem 279, 6190-6195. 
31. Persson IA, Josefsson M, Persson K, et al. (2006) Tea flavanols inhibit angiotensin-converting enzyme activity and increase nitric oxide production in human endothelial cells. J Pharm Pharmacol 58, 1139-1144.

32. Kim W, Jeong MH, Hong YJ, et al. (2006) The effect of green tea on endothelial dysfunction and circulating endothelial progenitor cells in the chronic smoker. Am J Cardiol 97, $60 \mathrm{D}$.

33. Nagaya N, Yamamoto H, Uematsu M, et al. (2004) Green tea reverses endothelial dysfunction in healthy smokers. Heart 90, $1485-1486$.

34. Widlansky ME, Hamburg NM, Anter E, et al. (2007) Acute EGCG supplementation reverses endothelial dysfunction in patients with coronary artery disease. J Am Coll Nutr 26, 95-102.

35. Murase T, Nagasawa A, Suzuki J, et al. (2002) Beneficial effects of tea catechins on diet-induced obesity: stimulation of lipid catabolism in the liver. Int J Obes 26, 1459-1464.

36. Wu LY, Juan CC, Ho LT, et al. (2004) Effect of green tea supplementation on insulin sensitivity in Sprague-Dawley rats. $J$ Agric Food Chem 52, 643-648.

37. Wolfram S, Raederstorff D, Preller M, et al. (2006) Epigallocatechin gallate supplementation alleviates diabetes in rodents. J Nutr 136, 2512-2518.

38. Anderson RA \& Polansky MM (2002) Tea enhances insulin activity. J Agric Food Chem 50, 7182-7186.

39. Broadhurst CL, Polansky MM \& Anderson RA (2000) Insulinlike biological activity of culinary and medicinal plant aqueous extracts in vitro. J Agric Food Chem 48, 849-852.

40. Chen PC, Wheeler DS, Malhotra V, et al. (2002) A green teaderived polyphenol, epigallocatechin-3-gallate, inhibits IкB kinase activation and IL- 8 gene expression in respiratory epithelium. Inflammation 26, 233-241.

41. Pan MH, Lin-Shiau SY, Ho CT, et al. (2000) Suppression of lipopolysaccharide-induced nuclear factor- $\kappa \mathrm{B}$ activity by theaflavin-3,3'-digallate from black tea and other polyphenols through down-regulation of ІкB kinase activity in macrophages. Biochem Pharmacol 59, 357-367.

42. Yang FJ, Oz HS, Barve S, et al. (2001) The green tea polyphenol (-)-epigallocatechin-3-gallate blocks nuclear factor-кB activation by inhibiting I $\mathrm{I} B$ kinase activity in the intestinal epithelial cell line IEC-6. Mol Pharmacol 60, 528-533.

43. Shoelson SE, Lee J \& Yuan M (2003) Inflammation and the $\mathrm{IKK} \beta / \mathrm{I} \kappa \mathrm{B} / \mathrm{NF}-\kappa \mathrm{B}$ axis in obesity- and diet-induced insulin resistance. Int J Obes $\mathbf{2 7}, \mathrm{S} 49-\mathrm{S} 52$.

44. Anton S, Melville L \& Rena G (2007) Epigallocatechin gallate (EGCG) mimics insulin action on the transcription factor FOXO1a and elicits cellular responses in the presence and absence of insulin. Cell Signal 19, 378-383.

45. Fukino Y, Shimbo M, Aoki N, et al. (2005) Randomized controlled trial for an effect of green tea consumption on insulin resistance and inflammation markers. J Nutr Sci Vitaminol 51, 335-342.

46. Ryu OH, Lee J, Lee KW, et al. (2006) Effects of green tea consumption on inflammation, insulin resistance and pulse wave velocity in type 2 diabetes patients. Diabetes Res Clin Prac 71, 356-358.

47. Venables MC, Hulston CJ, Cox HR, et al. (2008) Green tea extract ingestion, fat oxidation, and glucose tolerance in healthy humans. Am J Clin Nutr 87, 778-784.

48. Hursel R, Viechtbauer W \& Westerterp-Plantenga MS (2009) The effects of green tea on weight loss and weight maintenance: a meta-analysis. Int J Obes 33, 956-961.

49. Murase T, Misawa K, Haramizu S, et al. (2009) Catechininduced activation of the LKB1/AMP-activated protein kinase pathway. Biochem Pharmacol 78, 78-84.

50. Chen D, Wang CY, Lambert JD, et al. (2005) Inhibition of human liver catechol-O-methyltransferase by tea catechins and their metabolites: structure-activity relationship and molecular-modeling studies. Biochem Pharmacol 69, 1523-1531.

51. Wolfram S, Wang Y \& Thielecke F (2006) Anti-obesity effects of green tea: from bedside to bench. Mol Nutr Food Res 50, $176-187$

52. Wu AH, Tseng CC, Van den Berg D, et al. (2003) Tea intake, COMT genotype, and breast cancer in Asian-American women. Cancer Res 63, 7526-7529.

53. Sermswan A, Uboldejpracharak Y, Suthichaiyakul T, et al. (2002) Blood pressure response to antihypertensive agents related to baseline blood pressure. J Med Assoc Thai $\mathbf{8 5}$ 1113-1120. 